\title{
Endovascular Stroke Therapy Focused on Stent Retriever Thrombectomy and Direct Clot Aspiration : Historical Review and Modern Application
}

\author{
Dong-Hun Kang, M.D., ${ }^{1,2}$ Jaechan Park, M.D., Ph.D.' \\ Departments of Neurosurgery, Radiology, Kyungpook National University Hospital, Daegu-Gyeongbuk Cardiocerebrovascular Center, \\ School of Medicine, Kyungpook National University, Daegu, Korea
}

Intravenous recombinant tissue plasminogen activator had been the only approved treatment for acute ischemic stroke since its approval in 1995. However, the restrictive time window, numerous contraindications, and its low recanalization rate were all limitations of this modality. Under those circumstances, endovascular stroke therapy went through a great evolution during the past two decades of intravenous thrombolysis. The results of the 2013 randomized trials for endovascular stroke therapy were neutral, although they were limited by insufficient imaging screening at enrollment, early-generation devices with less efficacy, and treatment delays. Huge progress was made in 2015, as there were five randomized clinical trials which all demonstrated the safety and efficacy of endovascular stroke treatment. Despite differences in detail patient enrollment criteria, all 5 trials employed key factors for good functional recovery; (1) screening with non-invasive imaging to identify the proximal occlusion and exclude a large infarct core, (2) using highly effective modern thrombectomy devices mainly with stent retriever, and (3) establishment of a fast workflow to achieve effective reperfusion. The results of those trials indicate that modern thrombectomy devices can allow for faster and more effective reperfusion, which can lead to improved clinical outcomes compared to intravenous thrombolysis alone. These advances in mechanical thrombectomy are promising in the global fight against ischemic stroke-related disability and mortality. Two current mainstreams among such mechanical thrombectomy techniques, "stent retriever thrombectomy" and "direct clot aspiration", are the topic of this review. Stent retriever thrombectomy using Solitaire and Trevo retriever will be firstly discussed. And, the commonalities and the differences between two major clot aspiration thrombectomy techniques; a direct aspiration first pass technique (ADAPT) and forced arterial suction thrombectomy (FAST), will be additionally explained. Finally, details regarding the combination of direct clot aspiration and stent retriever thrombectomy, the switching strategy and the Solumbra technique, will be described.

Key Words : Acute ischemic stroke · Clot aspiration thrombectomy · Endovascular stroke therapy · Mechanical thrombectomy . Stent retriever thrombectomy.

\footnotetext{
- Received : September 26, 2016 •Revised : January 2, 2017 •Accepted : January 11, 2017

- Address for reprints : Jaechan Park, M.D., Ph.D.

Department of Neurosurgery, Kyungpook National University Hospital, Daegu-Gyeongbuk Cardiocerebrovascular Center, School of Medicine, Kyungpook National University, 130 Dongduk-ro, Jung-gu, Daegu 41944, Korea

Tel : +82-53-420-5647, Fax : +82-53-423-0504, E-mail : jparkmd@hotmail.com
}

This is an Open Access article distributed under the terms of the Creative Commons Attribution Non-Commercial License (http://creativecommons.org/licenses/by-nc/4.0) which permits unrestricted non-commercial use, distribution, and reproduction in any medium, provided the original work is properly cited. 


\section{INTRODUCTION}

Stroke is the second-leading global cause of death behind ischemic heart disease, occurring in an estimated 33 million people worldwide each year. Of the global deaths due to any stroke, ischemic stroke accounts for approximately 2.8 million deaths worldwide annually ${ }^{6,122}$. The first approved stroke treatment was intravenous recombinant tissue plasminogen activator (rt-PA); however, the efficacy was limited as a result of its narrow time window for application, certain contraindications, and low recanalization rates ${ }^{3,14,30,47)}$. This prompted an evolution in endovascular recanalization therapy during the past two decades, moving from intraarterial thrombolytics infusion to modern mechanical thrombectomy techniques. Among the various approaches, stent retriever thrombectomy and direct clot aspiration are two dominant methods in the present era. In this review, details of recent development regarding endovascular stroke therapy will be discussed with a focus on direct aspiration or clot aspiration thrombectomy.

\section{THE EVOLUTION OF MECHANICAL THROMBEC- TOMY FOR ACUTE STROKE MANAGEMENT : FIRST-GENERATION DEVICE AND TECHNIQUE}

Some of the earliest attempts at mechanical thrombectomy introduced mechanical clot disruption (MCD), which normally involved probing the thrombus with a microguidewire and/or a microcatheter. Recanalization was theoretically achieved by disrupting the main clot, but inevitably there were concerns related to distal clot embolization. Nevertheless, early results were promising ${ }^{33)}$. Modifications were then made to combine MCD with local intraarterial thrombolysis, which were shown to slightly improve overall recanalization rates ${ }^{37 \text {. }}$. Percutaneous balloon angioplasty was next considered as a type of clot disruption, as it involves advancement of a balloon mounted catheter to the occlusion site and inflation. Recanalization rates were further improved through the application of this technique. For example, in one period-to-period analysis comparing percutaneous balloon angioplasty and intraarterial thrombolysis-alone as a control, a Thrombolysis In Myocardial Infarction (TIMI) 2 or 3 recanalization rate was $91.2 \%$ in the percutaneous balloon angioplasty group as opposed to $63.9 \%$ in the intraarterial thrombolysis-alone group $(p<0.01)^{29)}$. One advanced form of clot disruption involved local application of ultrasound to disrupt a thrombus. The technique, named EKOS MicroLys US infusion catheter (EKOS Corporation, Bothell, WA, USA), was attempted in a small trial of 14 patients, and resulted in 57.1\% recanalization of TIMI 2 or $3^{28)}$. Notably, this method was included in the Interventional Management of Stroke (IMS) II trial, where it demonstrated a recanalization rate of $73 \%{ }^{20)}$. Another advanced form of clot disruption, called "endovascular photoacoustic recanalization” (EPAR; Endovasix Inc., Belmont, CA, USA), consisted in mechanical clot fragmentation by converting photonic energy from a laser into acoustic energy ${ }^{2}$.

The Merci retriever (Concentric Medical, Mountain View, CA, USA) was the first mechanical thrombectomy device to receive US Food and Drug Administration (FDA) approval. The Mechanical Embolus Removal in Cerebral Ischemia (MERCI) trial in 2005 demonstrated its effectiveness with recanalization rates significantly higher than in the historical control group ( $46 \%$ vs. $18 \%, p<0.0001)^{43)}$. Additionally, the multi-MERCI trial, which involved the second-generation Merci retriever, showed further improved recanalization rates $(57.3 \%)^{42}$. The next thrombectomy device to be approved was the Penumbra system (Penumbra, Alameda, CA, USA). The Penumbra pivotal stroke trial in 2009 demonstrated a successful revascularization rate of $81.6 \%$, for 125 eligible patients within an 8-hour time window from symptom onset ${ }^{35)}$.

\section{Stent retriever thrombectomy}

Stent retrievers or Stentrievers are self-expandable stent for thrombectomy that are deployed past the occlusion site with the use of an appropriate microcatheter, usually between 0.021- to 0.027-inch as inner diameter. The stent retriever is then expanded to capture the thrombus, which immediately may restore blood flow. And theoretically, such flow restoration can enhance the efficacy of systemic thrombolytic drugs if already in the circulation. After a period of up to $10 \mathrm{~min}$ utes, usually 3 to 5 minutes depending of the location and clot-size, the stent can be retrieved by pulling back the deployed stent into the guide catheter under proximal aspiration through the guide catheter. The addition of a proximal balloon guide catheter (BGC) can aid aspiration and help thrombus retrieval when the stent retriever is being dragged back into the guide catheter (Fig. 1).

The first stent retriever to be developed was Solitaire 


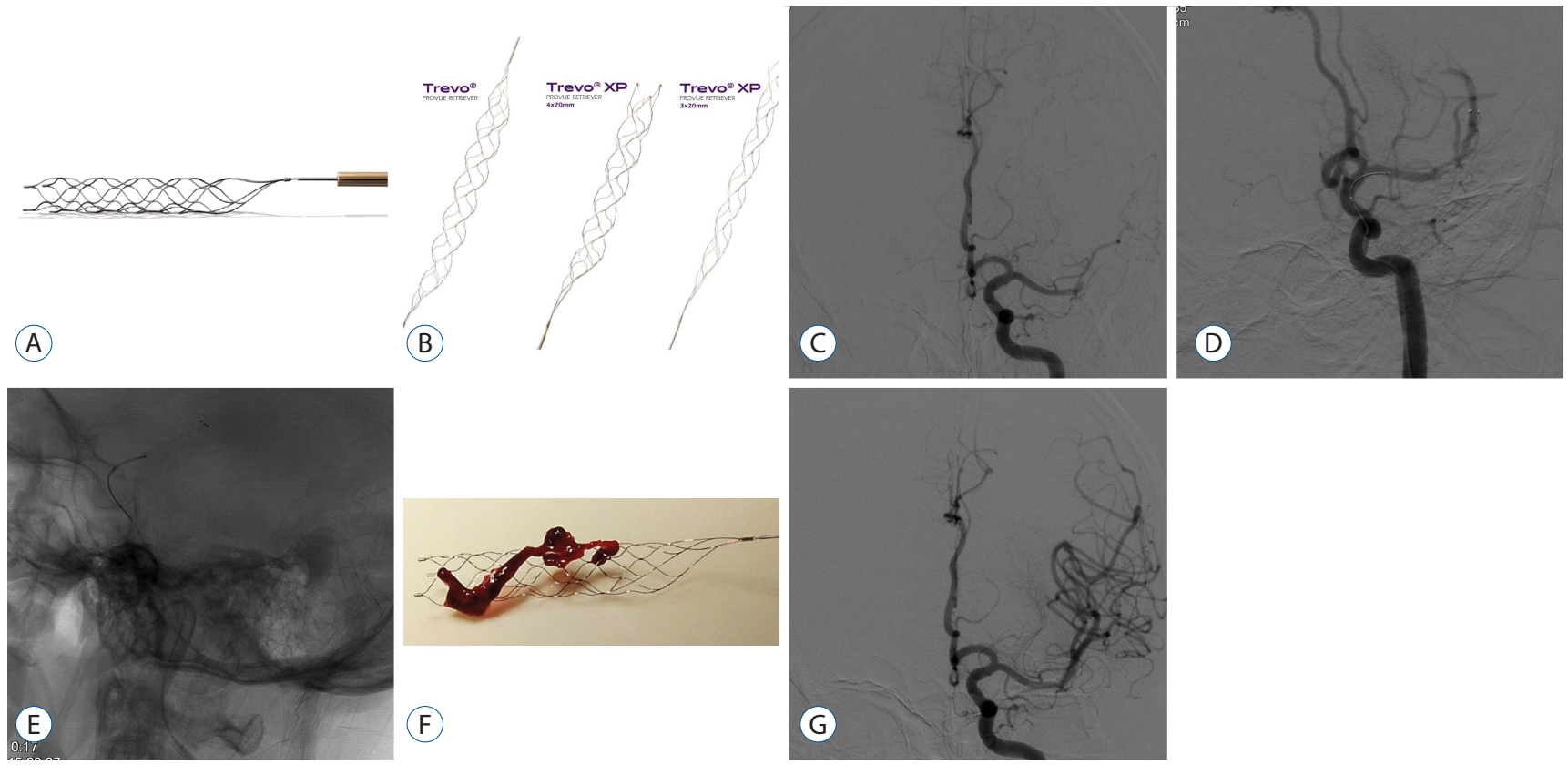

Fig. 1. A : Solitaire FR. B : Trevo retriever. A case example of stent retriever thrombectomy is the following. C : Baseline angiography shows total occlusion of the M1 segment of the left middle cerebral artery. D : Solitaire FR $4 \times 20$ is deployed across the thrombus and partial blood flow is immediately restored. E : After waiting for 3 minutes, the stent is retrieved by pulling back the stent into the guide catheter under proximal aspiration through the guide catheter. F and G : Final angiography shows full recanalization and the whole thrombus is retrieved within the stent-strut.

(Medtronic Neurovascular, Irvine, CA, USA), which was approved by FDA in 2012 and quickly became the first choice for many practitioners following the success of the Solitaire With the Intention For Thrombectomy (SWIFT) trial ${ }^{40)}$. The Solitaire FR can be fully deployed, fully resheathed, and recovered. The Solitaire is an FDA-approved stent retrieval device for use in ischemic stroke. Successful recanalization of TIMI grade 2 or 3 was achieved in $60.7 \%$ in the Solitaire group, but was only $24.1 \%$ in the Merci group. Good clinical outcome defined as modified Rankin Scale (mRS) 0 to 2 at 3 months $(58.2 \%$ vs. $33.6 \%)$ and mortality ( $17.2 \%$ vs. $38.2 \%)$ were favorable for the Solitaire stent as well, giving further positive evidence of mechanical thrombectomy devices.

The Trevo embolectomy device (Stryker Neurovascular, Fremont, CA, USA) is an alternative stent retriever device that can be advanced through a microcatheter past the occlusion site and deployed to cover the entire thrombus. Similar to the technique of Solitaire, the Trevo retriever is deployed for at least 5 to 10 minutes to ensure the clot is ensnared and flow is restored. After that, a BGC is inflated proximal to the clot to prevent anterograde flow, and the microcatheter and the embolectomy device are gently withdrawn through the guide catheter under continuous proximal aspiration. Trevo retriever has various line-ups and one distinguishing feature of this retriever from the generation of Trevo Provue is the fully visible stent-strut under fluoroscopy. The thrombectomy revascularisation of large vessel occlusions (TREVO) 2 trial demonstrated the success of the Trevo device in comparison to the Merci device in a similar fashion to the SWIFT trial. Recanalization rates with a Thrombolysis in Cerebral Ischemia (TICI) 2 or 3 were $86 \%$ in the Trevo group versus $60 \%$ in the Merci group, though there was no statistically significant difference regarding procedure-related adverse events between the two groups (15\% in the Trevo group vs. $23 \%$ in the Merci group, $p=0.18)^{32)}$.

\section{Step-by-step description of stent retriever throm- bectomy}

Regarding the guiding system for stent retriever thrombectomy, a large bore (8 or 9 French) BGC is preferred over a conventional guiding catheter, as the use of a BGC has been demonstrated to improve recanalization rate and clinical outcome by preventing distal embolization of captured clots during clot retrieval through the arrest of blood flow ${ }^{8,31)}$. 
However, BGC placement may not always easy if the stroke patient is elderly or a tortuous segment is encountered during the passage of aortic arch and/or common carotid artery. On those circumstances, a coaxial advancement technique can facilitate easier BGC placement compared to an exchange technique. For the coaxial technique, a 5 French $125 \mathrm{~cm}$ length angiography catheter is inserted through an 8 or 9 French BGC, and the relevant artery is catheterized using the inner 5 French angiography catheter. The angiography catheter is then advanced over a 0.035 inch guidewire, followed by a BGC. In some rare cases of extreme tortuosity, a triple coaxial technique; which is composed of a 5 French $125 \mathrm{~cm}$ length angiography catheter within a 100 to $115 \mathrm{~cm}$ length 6 French guiding catheter (Envoy or Envoy DA; Cordis Neurovascular, FL, US or Navien; Medtronic Neurovascular) within a 9 French BGC, is required to advance the guiding assembly.

Since a $4 \mathrm{~mm}$ diameter stent retriever is compatible with a 0.019 inch microcatheter, any type of microcatheter larger than 0.019 inch inner diameter can be used. However, a microcatheter with a 0.027 inch inner diameter is required when using a 5 or $6 \mathrm{~mm}$ stent retriever. The microcatheter is gently navigated beyond the occlusion using a 0.014 inch microguidewire. Then, a microangiography is performed to confirm that the microcatheter has been correctly located within the occluded parent artery. An appropriate-sized stent retriever is then deployed across the length of the occluded clot. After positioning the distal tip marker of the stent retriever, the stent retriever is carefully deployed by simultaneously pushing the delivery wire and unsheathing the microcatheter. This leads to better wall apposition of the struts of the stent retriever, which allows for better engagement of the clot and increases the chance of first-pass recanalization. Confirmation of proper stent position and restoration of the blood flow is evaluated by a control angiography immediately after deployment of the stent.

After waiting 3-5 minutes for stent retriever to engage the clot strongly, an additional control angiogram is obtained to check the surrounding status of stent retriever and the clot. Balloon of the BGC is then inflated to prevent distal clot migration during retrieval of the stent. When the stent and the microcatheter are retrieving simultaneously, the BGC needs to be continuously aspirated to prevent any distal embolization. In addition, the BGC still needs to be suctioned after retrieving the stent, until clear blood is aspirated without any clot debris. Finally, balloon of the BGC is deflated and a control angiography is obtained to evaluate the occlusion status.

\section{Stent retriever thrombectomy in difficult situa- tions}

In real practice, there can be several potentially difficult situations during the stent retriever thrombectomy procedure. First, the procedure can be difficult when the parent cerebral artery is very tortuous or includes a stenotic segment proximal to the occlusion ${ }^{23,38)}$. The advancement of a microcatheter and a stent retriever itself cannot be easy at the beginning of the procedure. Moreover, during the retrieval of the stent, the probability of losing the stent-engaged clots increases and continuous aspiration of the BGC may not effectively work to prevent distal embolization. In this case, the coaxial use of a 4 F or 5 F intermediate catheter (Navien; Medtronic Neurovascular or DAC; Stryker Neurovascular) or a large bore aspiration catheter (Penumbra reperfusion catheter; Penumbra) can increase the chance of clot retrieval. A large bore aspiration catheter or an intermediate catheter is coaxially introduced through the BGC and is advanced as close as possible to the stent-engaged clots. This helps to retain engaged clots when pulling the stent retriever back through a tortuous or stenotic segment.

A second difficult situation can develop with cervical carotid artery atherosclerotic disease. Cervical carotid artery atherosclerotic disease is responsible for approximately 15\% to $30 \%$ of acute ischemic strokes, which are mostly due to an artery-to-artery embolism with or without a cervical carotid artery occlusion ${ }^{15,25,34)}$. In the case of hemodynamic infarction, which can arise from a combination of acute cervical artery occlusion and poorly developed cross collaterals, the treatment strategy is straightforward to reopen the occluded cervical artery itself. However, if the cervical carotid artery causes tandem cervical and intracranial artery occlusions, which is more frequently met in the practice, it remains controversial whether to treat the intracranial artery occlusion or the cervical artery occlusion first ${ }^{41)}$. However, in the recent era of mechanical thrombectomy, the neurointerventionalists seem to favor treating the cervical artery occlusion first, followed by stent retriever thrombectomy for the tandem intracranial artery occlusion due to several reasons. First, the cervical internal carotid artery (ICA) must be sufficiently dilated for a stent 
retriever thrombectomy. Second reason is that a tandem intracranial occlusion can spontaneously dissolve after sufficient dilatation of the cervical ICA. Third, timely recanalization of the cervical ICA not only improves collateral flow to the penumbra, but also increases regional perfusion pressure and delivers fresh blood to the occlusion site to facilitate endogenous thrombolysis, which can possibly lead to recanalization after finishing the procedure ${ }^{44,49)}$.

Finally, the thrombectomy procedure can be complicated when it encounters large amount of clots in the cervical artery concomitantly with a tandem intracranial artery occlusion. In such cases, it is impossible to remove all the clots by only using a stent retriever. It can be more effective to perform a clot aspiration thrombectomy using a BGC and/or large bore catheter before stent retriever thrombectomy. If the proximal clot burden is very large, the BGC can be occluded during aspiration thrombectomy. A coaxial combination of an 8 F BGC within an $8 \mathrm{~F}$ shuttle sheath can be helpful to avoid the aforementioned situation. After recanalization of cervical artery using aspiration thrombectomy, a general stent retriever thrombectomy can be performed to remove any residual distal clots.

\section{RECENT RANDOMIZED CONTROLLED TRI- ALS OF ENDOVASCULAR STROKE THERAPY : THREE NEGATIVE, FIRST-GENERATION DEVICE TRIALS IN 2013 AND FIVE POSITIVE, STENT RETRIEVER TRIALS IN 2015}

Enthusiasm for mechanical thrombectomy was high after the SWIFT and TREVO-2 trials. However, this was dampened in 2013 when three separate randomized clinical trials revealed neutral results regarding endovascular therapy. First was IMS III, which compared endovascular treatment plus intravenous rt-PA versus intravenous rt-PA alone. As a result, the study found no significant benefits of endovascular treatment and was halted early due to its futility (90-day mRS 0-2, 40.8\% vs. $38.7 \%$, respectively) ${ }^{4)}$. Second, the MR RESCUE (Mechanical Retrieval and REcanalization of Stroke Clots Using Embolectomy) trial attempted to determine if imaging techniques could identify patients most likely to benefit from endovascular treatment and whether such treatment was superior to standard care. The results here were also neutral. Mean mRS was 3.9 in endovascular treatment group and also 3.9 in standard care group ${ }^{26)}$. Third, the SYNTHESIS Expansion trial randomly assigned 362 patients to endovascular therapy or intravenous rt-PA, but the results followed a pattern similar to the two previous trials ${ }^{9}$. Although the results of all the 2013 randomized trials were neutral, they were limited due to insufficient imaging screening, early-generation devices with less efficacy, and treatment delays ${ }^{36)}$. In 2015, a series of trials designed to overcome such limitations finally provided strong evidence of benefit from mechanical thrombectomy for acute stroke therapy. Table 1 summarizes the details.

Multicenter Randomized CLinical trial of Endovascular treatment for Acute ischemic stroke in the Netherlands (MR CLEAN ) was the first trial in 2015 to demonstrate the efficacy and safety of mechanical thrombectomy within a 6-hour time window from stroke onset. With a cohort of 500 patients, favorable outcome at 90 days occurred in 32.6\% of patients receiving endovascular treatment compared to $19.1 \%$ of patients receiving standard care alone. Also, there were no significant differences in mortality or symptomatic intracerebral hemorrhage $(\mathrm{ICH})^{1)}$. The second trial was EXtending the time for Thrombolysis in Emergency Neurological Deficits with IntraArterial therapy (EXTEND-IA), specifically designed for the Solitaire FR stent. The endovascular therapy group had a much higher incidence of favorable outcome as compared to patients receiving intravenous $\mathrm{rt}-\mathrm{PA}$ alone ( $\mathrm{mRS} 0-2$ at 90 days, $71 \%$ vs. $40 \%, p=0.01)^{5}$. The third positive result was the ESCAPE (Endovascular treatment for Small Core and Anterior circulation Proximal occlusion with Emphasis on minimizing CT to recanalization times) trial. Mechanical thrombectomy with available devices significantly outperformed standard care in efficacy (mRS 0-2 at 90 days, 53\% vs. 29.3\%, $p<0.001)^{13}$. The fourth trial was SWIFT PRIME (Solitaire With the Intention For Thrombectomy as PRIMary treatment for acute ischemic stroke). The study showed that for patients receiving intravenous rt-PA, thrombectomy with a stent retriever within a 6-hour time window resulted in improved functional outcomes (mRS $0-2$ at 90 days, $60 \%$ vs. $35 \%, p=0.001)^{39}$ ). The fifth positive trial was REVASCAT (Revascularization With Solitaire Device Versus Best Medical Therapy in Anterior Circulation Stroke Within 8 Hours). As with previous trials, this also demonstrated benefits of mechanical thrombectomy (mRS 0-2 at 90 days, $43.7 \%$ vs. $28.2 \%$, adjusted odds ratio 2.1 ). The rates of symptomatic ICH were $1.9 \%$ for both groups ${ }^{21)}$. 


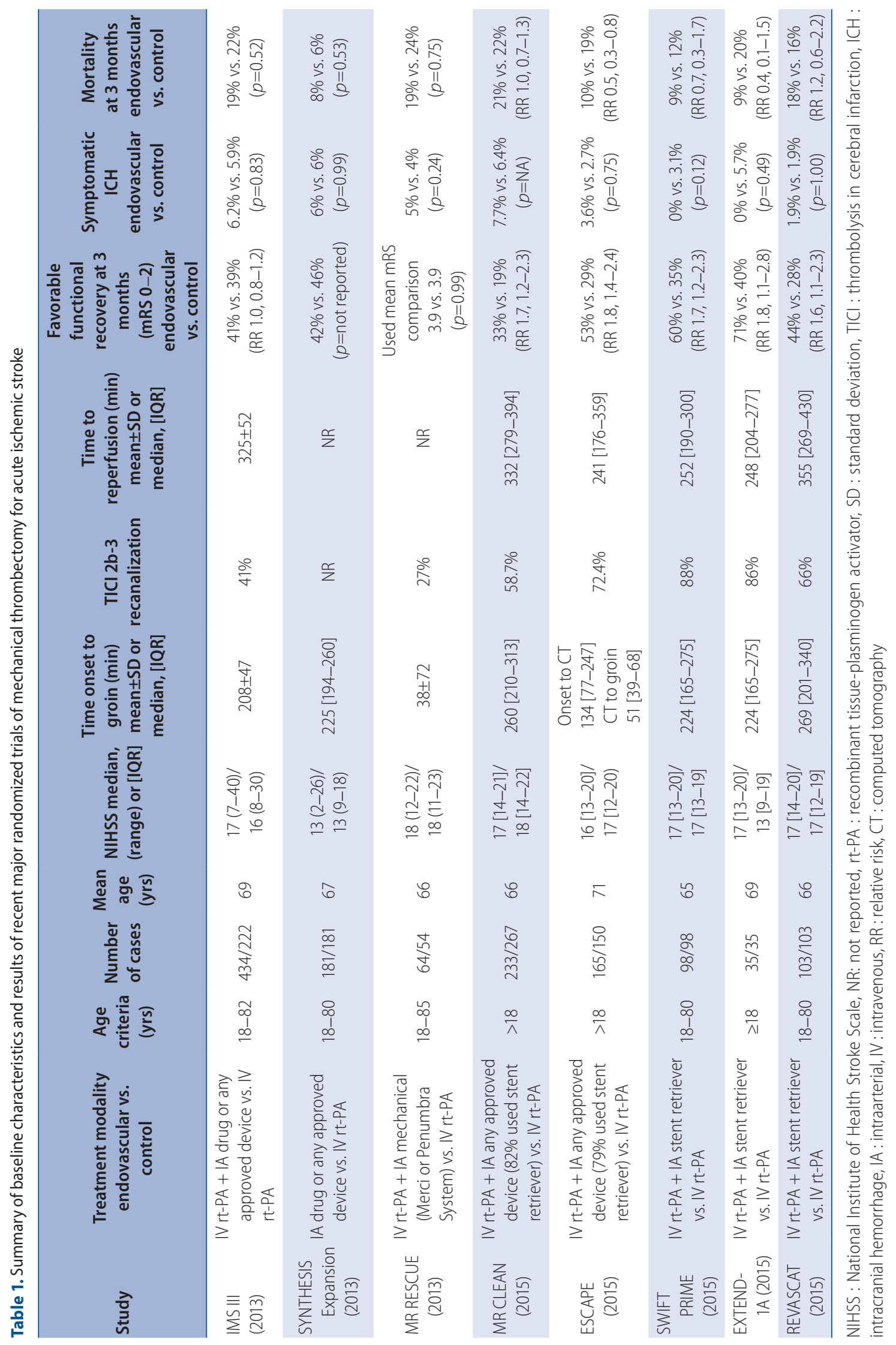




\section{CLOT ASPIRATION THROMBECTOMY FROM EARLIER ATTEMPTS TO ADAPT AND FAST US- ING THE PENUMBRA REPERFUSION CATHETER}

Clot aspiration thrombectomy had its origins in large, peripheral vessel manual aspiration techniques ${ }^{45}$. With respect to the technique, a large-bore catheter from 4 French to 8 French is advanced to the proximal surface of a clot and manual suction is applied using a syringe. The virtues of this method are it is inexpensive, fast to apply, and technically simple. Nevertheless, the application of the technique was restricted to non-tortuous vessels due to the large and stiff catheters available at the time ${ }^{7,19,48)}$. However, after the launching the Penumbra system (Penumbra), things began to change as the Penumbra reperfusion catheter had a large internal diameter that was not too stiff; therefore, it was very effective at advancement into intracranial vessels. This prompted practitioners to apply the Penumbra reperfusion catheter for direct clot aspiration of intracranial artery occlusions. Two examples of this new-generation clot aspiration technique were forced aspiration suction thrombectomy (FAST) and a direct aspiration first pass technique (ADAPT). The first version was FAST; and a couple of years later ADAPT followed ${ }^{24,46}$.

The modification in FAST was simple as the follows. The tip of the Penumbra reperfusion catheter was used as a vacuum pad for the thrombus, and forceful pulling of a syringe plunger allowed for intensified suction. This modified technique provided several benefits. First, as it was a simplification of the standard Penumbra system technique, no additional equipment was required. Second, revascularization can be achieved in an expedited manner, because the technique itself is brief. Third, if failure occurs, conversion to the standard Penumbra system technique or stent retriever thrombectomy is easy. The first case series on FAST was published in $2011^{24)}$. In that case series, 22 consecutive patients with large intracranial artery occlusion were enrolled; the locations were 14 on middle cerebral artery (MCA), 4 on ICA, and 4 on basilar artery (BA). After the FAST procedure, $81.9 \%$ of the patients had successfully recanalization of TICI $2 \mathrm{~b}(45.5 \%)$ or $3(36.4 \%)$. The favorable functional outcome at 3 months was $45.5 \%$. One year later, the authors of the first FAST paper reported the efficacy of FAST in acute distal ICA occlusions by comparing FAST and $\mathrm{MCD}^{18}$. In that analysis, the rates of TICI 2 or 3 recanalization and functional recovery at 3 months were significantly higher in the FAST group compared to the MCD group ( $85 \%$ vs. $32 \%$ and $45 \%$ vs. $16 \%$, respectively). Though this was a comparison between a new technique FAST and an outdated MCD at the time, the authors concluded FAST could be a viable option for acute distal ICA occlusion based on the high rate of successful recanalization and functional recovery.

In 2013, another new-version of clot aspiration, which modified the Penumbra system technique, took place called ADAPT. As discussed in that publication, ADAPT was very similar to the FAST technique. In that study, 37 consecutive cases were treated with the ADAPT technique, 30 of which involved the anterior circulation, seven involving the posterior. Revascularization was successful in all cases, including 65\% with complete recanalization (TICI 3$)^{46)}$.

\section{Difference between FAST and ADAPT}

The techniques of early FAST and ADAPT were similar in many aspects. Both mainly involved advancement of a largebore catheter to the occlusion site and a forceful aspiration thereafter. Regarding the differences between the two techniques, the authors of the ADAPT study pointed out that the FAST series involved an older generation aspiration catheter, such as the 041 and 032 family of Penumbra reperfusion catheters, as opposed to their more modern devices (Penumbra 5 Max) used two years after the FAST study. They mentioned two major advantages for using the new aspiration catheter for ADAPT. (1) The increased internal diameter allowed for an increased surface area, which provided better contact at the catheter tip-thrombus interface, and (2) the Penumbra 5 Max was more spacious in its proximal segment, which increased aspiration capacity $^{46)}$.

From the FAST author's standpoint, it was the additional use of a balloon guiding catheter (BGC) in subsequent years that set FAST slightly apart from ADAPT. The possible disadvantage of using a $\mathrm{BGC}$ is the time for preparation of a balloon in BGC. But there are several potential advantages here. The first is that using a BGC can minimize distal clot migration or embolization. For example, in an in vitro study published in 2013, the Merci retriever, Solitaire FR, and Trevo devices were compared with and without the use of a BGC to ascertain the number of distal emboli generated during thrombus retrieval. They found that the use of the BGC during thrombus retrieval significantly reduced the formation of large distal emboli with a diameter $>1 \mathrm{~mm}$, regardless of the 
device used $(p<0.01)^{8)}$. That brings up a second potential advantage. It may be the case that using a BGC might enhance aspiration efficacy during the FAST procedure by blocking proximal flow. Because the distal part is already blocked by the clot, proximal occlusion with a BGC can make a vacuum there, which then may make the negative pressure more effective. But this is a theoretical point; further in vitro confirmation seems necessary. The third advantage is that it can allow for prompt switching to stent retriever technique in case of failure with FAST. This may be important, especially in light of modern trials, because all the large-volume stent retriever trials recommend using the BGC, for example, SWIFT and SWIFT PRIME ${ }^{39,40)}$.

\section{Step-by-step description of the FAST technique (Fig. 2)}

The FAST procedure begins with femoral puncture to the mid-portion of the common femoral artery. The combination of intravenous rt-PA and mechanical thrombectomy with an
8 or 9 French guiding catheter, and correspondingly large femoral sheaths, can increase the risk of significant groin complications. Therefore, safe femoral puncture importantly counts in preventing such events. The next step is advancing the guiding catheter to the proximity of the occluded target vessel. Common selections for a guiding catheter during the FAST technique include either an 8 French or a 9 French BGC. Specifically, the author mostly used a 9 French BGC, either an Optimo (Tokai Medical Products, Aichi, Japan) or a Merci (Stryker Neurovascular). For the cases of vertebral artery which is not allowed the BGCs due to small caliber, any general 6 French guide catheter can be used as a substitute.

Once the BGC is optimally positioned, the microcatheter and microguidewire are advanced through it up to the occlusion. It is recommended that a 2.0 French or a 2.3 French microcatheter, with a 0.014 inch or a 0.016 inch microguidewire inside it, is introduced into a large-bore aspiration catheter. This set of construct is introduced into the BGC and is advanced to the occlusion site. During this stage, it is recommended to avoid distal passage of the thrombus by a micro-
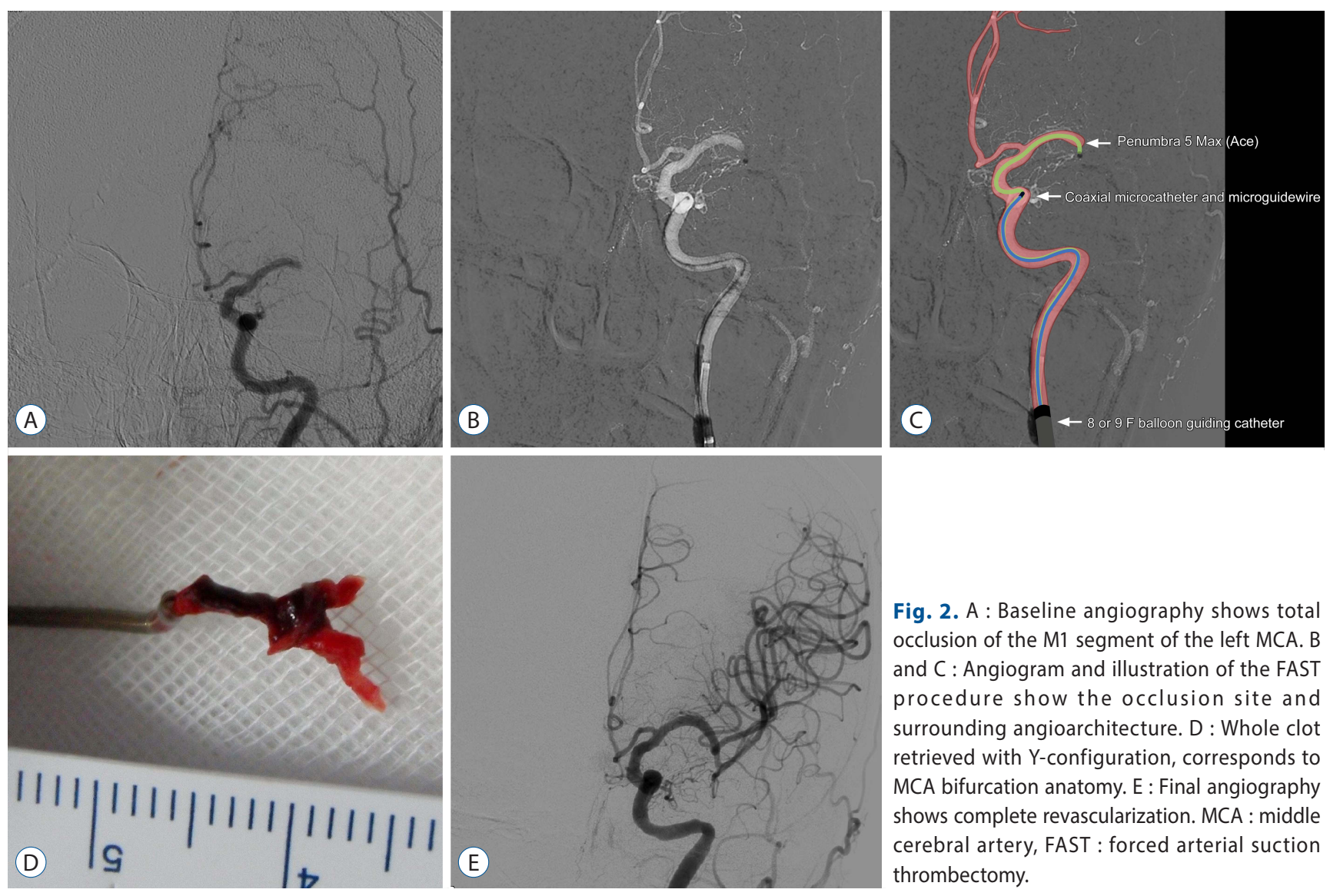

Fig. 2. A : Baseline angiography shows total occlusion of the M1 segment of the left MCA. B and $C$ : Angiogram and illustration of the FAST procedure show the occlusion site and surrounding angioarchitecture. D : Whole clot retrieved with $\mathrm{Y}$-configuration, corresponds to MCA bifurcation anatomy. $E$ : Final angiography shows complete revascularization. MCA : middle cerebral artery, FAST : forced arterial suction thrombectomy. 
catheter or a microguidewire, because distal passage itself can theoretically elevate the chance of clot disruption and migration. Therefore, in cases where the patient's vascular anatomy allows, the large-bore aspiration catheter is directly advanced to the thrombus without any distal passage of the clot by an inner microcatheter or a microguidewire. However, in the cases where a patient's arterial anatomy is tortuous, an inner microcatheter is necessarily advanced past the thrombus over a microguidewire. Then, the aspiration catheter is advanced over it up to the thrombus. Local angiography can be performed before aspiration is done to predict the original path of the occluded segment and to outline the occlusion. Once the large-bore aspiration catheter is located optimally, the inner microcatheter and microguidewire are then removed. Thereafter, the proximal hub of the aspiration catheter is directly connected to a $20 \mathrm{~mL}$ or $50 \mathrm{~mL}$ syringe. Slight negative pressure is attempted by partial pulling of the plunger to confirm direct wedging and a vacuum state between the tip of the large-bore aspiration catheter and the clot. Absence of backflow mostly indicates the thrombus is trapped in the catheter. Just before applying the negative pressure, the balloon of the BGC is inflated to block proximal flow. Then negative pressure applied by forceful pulling of the plunger of the syringe for a period of 60-90 seconds. After that, the aspiration catheter is slightly advanced further into the thrombus and then slowly withdrawn under continuous aspiration. If the vacuum state is unexpectedly lifted, followed by entry of free blood flow into the catheter, this suggests the wedged clot is disrupted and sucked into the catheter. Or if the wedging or vacuum state persists, so no free blood flow in the syringe tubing is restored upon withdrawing the large-bore aspiration catheter into the BGC, the aspiration catheter is slowly and carefully removed from the patient under continuous aspiration. And the BGC is manually aspirated with a syringe to remove any residual thrombus fragments. Finally, the balloon of the BGC is slowly deflated to restore proximal blood flow. This process can be repeated until successful reperfusion is achieved. However, if three FAST passes fail to achieve incremental reperfusion, then stent retriever thrombectomy may be employed.

\section{Technical tips to overcome tortuous segment during the FAST}

Despite huge advances in the aspiration catheter's tracking ability, tortuosity itself and underlying atherosclerotic stenosis remain obstacles for passage. Moreover, the anatomic condition where a small artery is arising from a tortuous segment, such as an ophthalmic artery origin from the carotid siphon, can be an additional challenge. The technical tips to overcome the aforementioned problem are as follows.

The first tip is that using the largest and simultaneously softest aspiration catheter on the practitioner's hand and maximizing guiding catheter support. This simply allows better control of aspiration catheter by the practitioner. The second is steam shaping of the tip of the Penumbra reperfusion catheter (Fig. 3). If some curves are provided to the tip of the large-bore catheters, it theoretically helps to pass the tortuous segment during the FAST procedure. This can be achieved through steam shaping. Three grades of "simple-curved" steam shaping are recommended. In most of the cases, advancement is more smoothly achieved by steam shaping the tip of the catheter with a $45^{\circ}$ curve. However, in some proportion of the cases with extreme tortuosity or severe atherosclerotic stenosis, a $90^{\circ}$ curve or J-shape can be used. The third tip to overcome tortuosity is the coaxial advancement technique
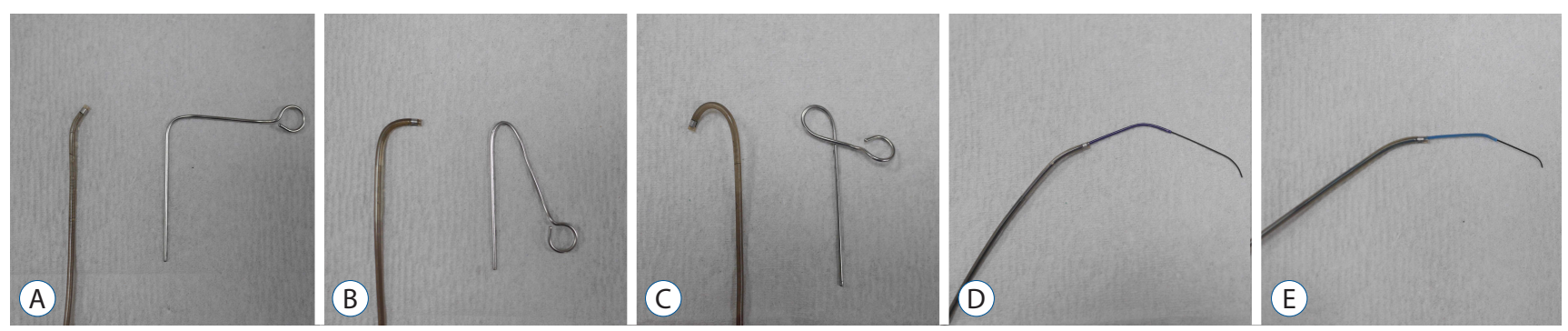

Fig. 3. A : Examples of steam shaping of the catheter tip with $45^{\circ}$ curve. $B: 90^{\circ}$ curve. $C$ : J shape. D : Examples of coaxial advancement technique are the following. Penumbra 5 Max or 5 Max Ace is assembled with a 2.3 French inner microcatheter and a 0.016 inch microguidewire. E : Penumbra 4 Max is assembled with a 2.0 French inner microcatheter and a 0.014 inch microguidewire. 
(Fig. 3). If a microguidewire is just used for advancing the Penumbra aspiration catheter, the gap between the two devices will be large. This can result in difficulty steering and controlling the catheter, thus the advancement through a highly curved segment is technically unlikely. The coaxial advancement technique is recommended here, which means introducing another microcatheter of intermediate size between the aspiration catheter and microguidewire to reduce the gap between them. This serves to restrict the freedom of movement of the Penumbra reperfusion catheter, which will allow greater ability to steer and control during advancement. The author's technical 'recipe for coaxial assembly' is as follows (Fig. 4). For the ICA and the proximal M1 segment of MCA, it is recommended to use the Penumbra 5 Max or 5 Max Ace with a 2.3 French inner microcatheter (Prowler Select Plus; Cordis Neurovascular), and a 0.016 inch microguidewire (GT; Terumo, Tokyo, Japan). For the distal M1 segment or the M2 segment of MCA, the Penumbra 4 Max with a 2.0 French inner microcatheter (Excelsior 1018; Stryker Neurovascular), and a 0.014 inch microguidewire (Synchro; Stryker Neurovascular) are recommended.

\section{Penumbra reperfusion catheters and other next- generation catheters for aspiration}

The efficacy of clot aspiration thrombectomy is strongly associated with the force of aspiration at the tip of the large-bore aspiration catheter. Therefore, differences in the properties of each catheter may inevitably determine different aspiration forces and the efficacy of the procedure. In 2014, one in vitro study compared various parameters of four large-bore catheters commercially available at the time ${ }^{16)}$. They analyzed catheter tip force, aspiration flow rate, and effective flow lumen to determine the optimal catheter for clot aspiration thrombectomy based on hemodynamic background. The study demon-
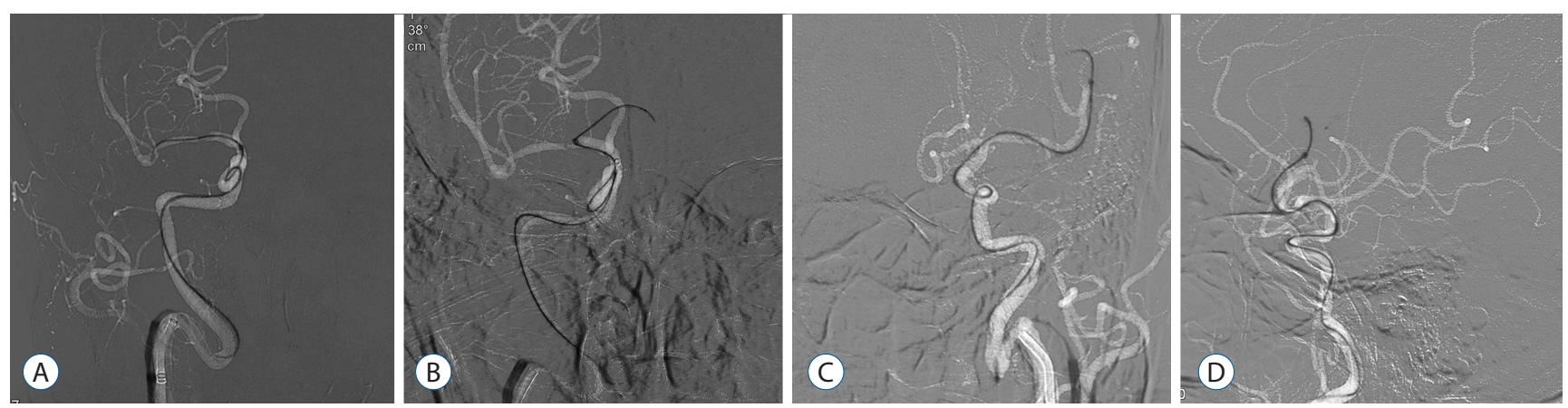

Fig. 4. Examples of how steam shaping and coaxial assembly used during the FAST procedure. A : Penumbra 5 Max Ace is advancing to M1 segment of MCA in a patient of acute ICA terminus occlusion on the right. B: Penumbra 5 Max Ace is then advancing to A1 segment of ACA in the same patient. $C$ and $D$ : Penumbra 4 Max is introducing to the left M2 segment in a patient of acute occlusion at M2 segment of MCA on the left. FAST : forced arterial suction thrombectomy, MCA : middle cerebral artery, ICA : internal carotid artery, ACA : anterior cerebral artery.

Table 2. Specification of the new generation large-bore catheters for both clot aspiration and providing stability

\begin{tabular}{lccccc}
\hline Product name (company) & Length $(\mathbf{c m})$ & $\begin{array}{c}\text { Proximal OD } \\
\text { (inches) }\end{array}$ & $\begin{array}{c}\text { Proximal ID } \\
\text { (inches) }\end{array}$ & Distal OD (inches) & Distal ID (inches) \\
\hline Ace 64 (Penumbra) & 132 & 0.080 & 0.068 & 0.075 & 0.064 \\
Arc (Medtronic neurovascular) & 132 & 0.080 & 0.069 & 0.069 & 0.061 \\
Catalyst (Stryker neurovascular) & 132 & 0.079 & 0.060 & 0.071 & 0.060 \\
Sofia (Microvention) & 125 & 0.068 & 0.055 & 0.068 & 0.055 \\
Sofia plus (Microvention) & $125 / 131$ & 0.083 & 0.056 & 0.065 & 0.070 \\
Revive IC 056 (Cordis neurovascular) & 121 & 0.065 & & 0.056 \\
\hline
\end{tabular}

$\mathrm{OD}$ : outer diameter, ID : inner diameter 
strated that the Penumbra 5 Max Ace catheter outperformed the other catheters regarding the aforementioned hemodynamic properties, perhaps due to its larger lumen and tapered design. Recently there have been several new large-bore catheters that have just started to be commercially available (Table 2). These include the Ace 064 (Penumbra), Arc (Medtronic Neurovascular), Catalyst (Stryker Neurovascular), Revive IC (Cordis Neurovascular), and Sofia (MicroVention, Tustin, CA, USA). Therefore, additional in vitro study to compare hemodynamic parameters of the new catheters seems required. And more importantly, the accumulation of experience and clinical data from real practice is strongly required with regard to the evaluation of new devices.

\section{COMBINED USAGE OF DIRECT CLOT ASPIRATION AND STENT RETRIEVER THROMBECTOMY : THE SWITCHING STRATEGY AND THE SOLUMBRA TECHNIQUE}

There are two dominant mechanical thrombectomy techniques in the present era, which are stent retriever thrombectomy and direct clot aspiration. Additionally, there have been a few attempts to enhance the rate of successful recanalization through a combination of stent retriever thrombectomy and direct clot aspiration. The first is called the "switching strategy," which involves switching from FAST to Solitaire stent thrombectomy ${ }^{22)}$ and the second is the "Solumbra technique," which uses the two devices simultaneously ${ }^{11,17,27)}$.

Although both attempts bear similarities in terms of the concept of combined usage, the details are very different. The "switching strategy" attempts to achieve successful recanalization by using only one device, and then "switch" to a second device as a rescue procedure. A period-to-period comparison analysis for introducing the switching strategy was published in $2013^{22)}$. In period 1, the investigators used FAST only for recanalization; however in period 2, they attempted a "switching strategy" from FAST to the stent retriever in difficult cases. In the latter period, they hypothesized additional attempts with a different mechanism of Solitaire stent could improve recanalization in difficult cases with FAST. Resultantly, patients in period 2 showed a trend for higher TICI $2 \mathrm{~b}$ or 3 recanalization (73.8\% vs. $85.1 \%, p=0.10)$ and a significantly better 3 -month functional outcome ( $49.2 \%$ vs. $67.6 \%, p=0.03)$.
On the other hand, the "Solumbra technique" simultaneously uses both the stent retriever and the large-bore aspiration catheter at once to enhance the efficacy of recanalization. The most common combination is to use the Solitaire FR (Medtronic Neurovascular) and the Penumbra reperfusion catheter (Penumbra), so this has come to be known as Solumbra. The users hypothesized that localized aspiration at the site of the thrombus may promote entrapment of the thrombus within the stent. Also, flow control induced by aspiration may reduce the incidence of thrombus fragmentation and distal embolization. There has been a number of case series and clinical studies associated with good result of the Solumbra technique $^{11,17,27)}$. However, it is still an area of controversy whether the Solumbra technique is more beneficial when compared with the present major thrombectomy techniques ${ }^{10)}$.

\section{CONCLUSION}

Mechanical thrombectomy is a landmark change in stroke management, and guidelines should recommend mechanical thrombectomy as a level 1 evidence-based treatment worldwide. In terms of technical aspects of mechanical thrombectomy, stent retrievers (Solitaire FR or Trevo device) as the primary method and clot aspiration thrombectomy (FAST or ADAPT) as a solid secondary rescue method will be the trend for some time. Moreover, the efficacy of combined usage of both stent retriever and clot aspiration is continuously improving due to recent development in device technology. Therefore, it seems necessary for the practitioner to learn how to perform stent retriever thrombectomy, clot aspiration thrombectomy, as well as a combined usage of both, whether choosing the switching strategy or the Solumbra technique.

\section{References}

1. Berkhemer OA, Fransen PS, Beumer D, van den Berg LA, Lingsma HF, Yoo AJ, et al.; MR CLEAN Investigators : a randomized trial of intraarterial treatment for acute ischemic stroke. N Engl J Med 372 : 11-20, 2015

2. Berlis $A$, Lutsep $H$, Barnwell $S$, Norbash $A$, Wechsler $L$, Jungreis $C A$, et al. : Mechanical thrombolysis in acute ischemic stroke with endovascular photoacoustic recanalization. Stroke 35 : 1112-1116, 2004

3. Brandt T, von Kummer R, Müller-Küppers $M$, Hacke W : Thrombolytic 
therapy of acute basilar artery occlusion. Variables affecting recanalization and outcome. Stroke 27 : 875-881, 1996

4. Broderick JP, Palesch YY, Demchuk AM, Yeatts SD, Khatri P, Hill MD, et al.; Interventional Management of Stroke (IMS) III Investigators : Endovascular therapy after intravenous t-PA versus t-PA alone for stroke. $\mathbf{N}$ Engl J Med 368 : 893-903, 2013

5. Campbell BC, Mitchell PJ, Kleinig TJ, Dewey HM, Churilov L, Yassi N, et al.; EXTEND-IA Investigators : Endovascular therapy for ischemic stroke with perfusion-imaging selection. N Engl J Med 372 : 1009-1018, 2015

6. Caplan LR, Wityk RJ, Glass TA, Tapia J, Pazdera L, Chang HM, et al. : New England Medical Center Posterior Circulation registry. Ann Neurol $56:$ 389-398, 2004

7. Chapot R, Houdart E, Rogopoulos A, Mounayer C, Saint-Maurice JP, Merland JJ : Thromboaspiration in the basilar artery: report of two cases. AJNR Am J Neuroradiol 23 : 282-284, 2002

8. Chueh JY, Kühn AL, Puri AS, Wilson SD, Wakhloo AK, Gounis MJ : Reduction in distal emboli with proximal flow control during mechanical thrombectomy: a quantitative in vitro study. Stroke 44 : 1396-1401, 2013

9. Ciccone A, Valvassori L, Nichelatti M, Sgoifo A, Ponzio M, Sterzi R, et al.; SYNTHESIS Expansion Investigators : Endovascular treatment for acute ischemic stroke. N Engl J Med 368 : 904-913, 2013

10. Delgado Almandoz JE, Kayan Y, Young ML, Fease JL, Scholz JM, Milner AM, et al. : Comparison of clinical outcomes in patients with acute ischemic strokes treated with mechanical thrombectomy using either Solumbra or ADAPT techniques. J Neurointerv Surg 8 : 1123-1128, 2016

11. Deshaies EM : Tri-axial system using the Solitaire-FR and Penumbra Aspiration Microcatheter for acute mechanical thrombectomy. J Clin Neurosci 20 : 1303-1305, 2013

12. Feigin VL, Forouzanfar MH, Krishnamurthi R, Mensah GA, Connor M, Bennett DA, et al.; Global Burden of Diseases, Injuries, and Risk Factors Study 2010 (GBD 2010) and the GBD Stroke Experts Group : Global and regional burden of stroke during 1990-2010: findings from the Global Burden of Disease Study 2010. Lancet 383 : 245-254, 2014

13. Goyal M, Demchuk AM, Menon BK, Eesa M, Rempel JL, Thornton J, et al.; ESCAPE Trial Investigators : Randomized assessment of rapid endovascular treatment of ischemic stroke. N Engl J Med 372 : 1019-1030, 2015

14. Hacke W, Zeumer H, Ferbert A, Brückmann H, del Zoppo GJ : Intraarterial thrombolytic therapy improves outcome in patients with acute vertebrobasilar occlusive disease. Stroke 19 : 1216-1222, 1988

15. Holmstedt CA, Turan TN, Chimowitz MI : Atherosclerotic intracranial arterial stenosis: risk factors, diagnosis, and treatment. Lancet Neurol 12 : 1106-1114, 2013

16. Hu YC, Stiefel MF : Force and aspiration analysis of the ADAPT technique in acute ischemic stroke treatment. J Neurointerv Surg 8 : 244246, 2016

17. Humphries W, Hoit D, Doss VT, Elijovich L, Frei D, Loy D, et al. : Distal aspiration with retrievable stent assisted thrombectomy for the treatment of acute ischemic stroke. J Neurointerv Surg 7 : 90-94, 2015
18. Hwang YH, Kang DH, Kim YW, Kim YS, Park SP, Suh CK : Outcome of forced-suction thrombectomy in acute intracranial internal carotid occlusion. J Neurointerv Surg 5 Suppl 1 : i81-i84, 2013

19. Imai K, Mori T, Izumoto H, Takabatake N, Kunieda T, Shimizu H, et al. : Clot removal therapy by aspiration and extraction for acute embolic carotid occlusion. AJNR Am J Neuroradiol 27 : 1521-1527, 2006

20. IMS II Trial Investigators : The interventional management of stroke (IMS) II study. Stroke 38 : 2127-2135, 2007

21. Jovin TG, Chamorro A, Cobo E, de Miquel MA, Molina CA, Rovira A, et al.; REVASCAT Trial Investigators : Thrombectomy within 8 hours after symptom onset in ischemic stroke. N Engl J Med 372 : 2296-2306, 2015

22. Kang DH, Kim YW, Hwang YH, Park J, Hwang JH, Kim YS : Switching strategy for mechanical thrombectomy of acute large vessel occlusion in the anterior circulation. Stroke $44:$ 3577-3579, 2013

23. Kang DH, Kim YW, Hwang YH, Park SP, Kim YS, Baik SK : Instant reocclusion following mechanical thrombectomy of in situ thromboocclusion and the role of low-dose intra-arterial tirofiban. Cerebrovasc Dis 37 : 350-355, 2014

24. Kang DH, Hwang YH, Kim YS, Park J, Kwon O, Jung C : Direct thrombus retrieval using the reperfusion catheter of the penumbra system: forcedsuction thrombectomy in acute ischemic stroke. AJNR Am J Neuroradiol 32 : 283-287, 2011

25. Kasner SE, Chimowitz MI, Lynn MJ, Howlett-Smith H, Stern BJ, Hertzberg VS, et al. : Predictors of ischemic stroke in the territory of a symptomatic intracranial arterial stenosis. Circulation 113 : 555-563, 2006

26. Kidwell CS, Jahan R, Gornbein J, Alger JR, Nenov V, Ajani Z, et al.; MR RESCUE Investigators : A trial of imaging selection and endovascular treatment for ischemic stroke. N Engl J Med 368 : 914-923, 2013

27. Lee JS, Hong JM, Lee SJ, Joo IS, Lim YC, Kim SY : The combined use of mechanical thrombectomy devices is feasible for treating acute carotid terminus occlusion. Acta Neurochir (Wien) 155 : 635-641, 2013

28. Mahon BR, Nesbit GM, Barnwell SL, Clark W, Marotta TR, Weill A, et al. : North American clinical experience with the EKOS MicroLysUS infusion catheter for the treatment of embolic stroke. AJNR Am J Neuroradiol 24 : 534-538, 2003

29. Nakano S, Iseda T, Yoneyama T, Kawano H, Wakisaka S : Direct percutaneous transluminal angioplasty for acute middle cerebral artery trunk occlusion: an alternative option to intra-arterial thrombolysis. Stroke 33 : 2872-2876, 2002

30. National Institute of Neurological Disorders and Stroke rt-PA Stroke Study Group : Tissue plasminogen activator for acute ischemic stroke. N Engl J Med 333 : 1581-1587, 1995

31. Nguyen TN, Malisch T, Castonguay AC, Gupta R, Sun CH, Martin CO, et al. : Balloon guide catheter improves revascularization and clinical outcomes with the Solitaire device: analysis of the North American Solitaire Acute Stroke Registry. Stroke 45 : 141-145, 2014

32. Nogueira RG, Lutsep HL, Gupta R, Jovin TG, Albers GW, Walker GA, et al.; TREVO 2 Trialists : Trevo versus Merci retrievers for thrombectomy revascularisation of large vessel occlusions in acute ischaemic stroke (TREVO 2): a randomised trial Lancet 380 : 1231-1240, 2012 
33. Noser EA, Shaltoni HM, Hall CE, Alexandrov AV, Garami Z, Cacayorin $E D$, et al. : Aggressive mechanical clot disruption: a safe adjunct to thrombolytic therapy in acute stroke? Stroke $36: 292-296,2005$

34. Papanagiotou P, Roth C, Walter S, Behnke S, Grunwald IQ, Viera J, et al. : Carotid artery stenting in acute stroke. J Am Coll Cardiol 58 : 23632369, 2011

35. Penumbra Pivotal Stroke Trial Investigators : The penumbra pivotal stroke trial: safety and effectiveness of a new generation of mechanical devices for clot removal in intracranial large vessel occlusive disease. Stroke 40 : 2761-2768, 2009

36. Qureshi Al, Abd-Allah F, Aleu A, Connors JJ, Hanel RA, Hassan AE, et al. : Endovascular treatment for acute ischemic stroke patients: implications and interpretation of IMS III, MR RESCUE, and SYNTHESIS EXPANSION trials: a report from the Working Group of International Congress of Interventional Neurology. J Vasc Interv Neurol 7 : 56-75, 2014

37. Qureshi Al, Siddiqui AM, Suri MF, Kim SH, Ali Z, Yahia AM, et al. : Aggressive mechanical clot disruption and low-dose intra-arterial thirdgeneration thrombolytic agent for ischemic stroke: a prospective study. Neurosurgery 51 : 1319-1327; discussion 1327-1329, 2002

38. Ryu CW, Kwak HS, Jahng GH, Lee HN : High-resolution MRI of intracranial atherosclerotic disease. Neurointervention 9 : 9-20, 2014

39. Saver JL, Goyal M, Bonafe A, Diener HC, Levy El, Pereira VM, et al.; SWIFT PRIME Investigators : Stent-retriever thrombectomy after intravenous t-PA vs. t-PA alone in stroke. $\mathbf{N}$ Engl J Med 372 : 2286-2295, 2015

40. Saver JL, Jahan R, Levy El, Jovin TG, Baxter B, Nogueira RG, et al.; SWIFT Trialists : Solitaire flow restoration device versus the Merci Retriever in patients with acute ischaemic stroke (SWIFT): a randomised, parallelgroup, non-inferiority trial. Lancet 380 : 1241-1249, 2012

41. Sivan-Hoffmann R, Gory B, Armoiry X, Goyal M, Riva R, Labeyrie PE, et al. : Stent-retriever thrombectomy for acute anterior ischemic stroke with tandem occlusion: a systematic review and meta-analysis. Eur Radiol 27 : 247-254, 2017

42. Smith WS, Sung G, Saver J, Budzik R, Duckwiler G, Liebeskind DS, et al. : Mechanical thrombectomy for acute ischemic stroke: final results of the Multi MERCI trial. Stroke 39 : 1205-1212, 2008

43. Smith WS, Sung G, Starkman S, Saver JL, Kidwell CS, Gobin YP, et al. : Safety and efficacy of mechanical embolectomy in acute ischemic stroke: results of the MERCI trial. Stroke 36 : 1432-1438, 2005

44. Stampfl S, Ringleb PA, Möhlenbruch M, Hametner C, Herweh C, Pham M, et al. : Emergency cervical internal carotid artery stenting in combination with intracranial thrombectomy in acute stroke. AJNR Am J Neuroradiol 35 : 741-746, 2014

45. Starck EE, McDermott JC, Crummy AB, Turnipseed WD, Acher CW, Burgess $\mathrm{JH}$ : Percutaneous aspiration thromboembolectomy. Radiology $156: 61-66,1985$

46. Turk AS, Spiotta A, Frei D, Mocco J, Baxter B, Fiorella D, et al. : Initial clinical experience with the ADAPT technique: a direct aspiration first pass technique for stroke thrombectomy. J Neurointerv Surg 6 : 231237, 2014

47. Wardlaw JM, Murray V, Berge E, del Zoppo G, Sandercock P, Lindley RL, et al. : Recombinant tissue plasminogen activator for acute ischaemic stroke: an updated systematic review and meta-analysis. Lancet 379 : 2364-2372, 2012

48. Xu GF, Suh DC, Choi CG, Kim JK, Kim W, Kim SJ, et al. : Aspiration thrombectomy of acute complete carotid bulb occlusion. J Vasc Interv Radiol 16 : 539-542, 2005

49. Yoon W, Kim BM, Kim DJ, Kim DI, Kim SK : Outcomes and prognostic factors after emergent carotid artery stenting for hyperacute stroke within 6 hours of symptom onset. Neurosurgery 76 : 321-329, 2015 